\title{
Next generation reactive transport models
}

\section{Journal Article}

Author(s):

Vrijmoed, Johannes C.; Podladchikov, Yuri Y.; Malvoisin, Benjamin

Publication date:

2015

Permanent link:

https://doi.org/10.3929/ethz-a-010736969

Rights / license:

In Copyright - Non-Commercial Use Permitted

\section{Originally published in:}

Geotectonic Research 97(1), https://doi.org/10.1127/1864-5658/2015-37

Funding acknowledgement:

335577 - Interplay between metamorphism and deformation in the Earth's lithosphere (EC) 
Vrijmoed, J. C. ${ }^{1}$, Podladchikov, Y, Y. ${ }^{2}$, and Malvoisin, B. ${ }^{1,2}$

1 Institute of Geochemistry and Petrology, ETH Zurich, Switzerland

2 Institute des Sciences de la Terre, UNIL, Switzerland

3 Centre d’Hydrogéology et de Géothermie, Université de Neuchâtel, Neuchâtel, Switzerland

\section{Introduction}

The interaction between deformation, fluid flow and reaction plays a fundamental role in geological processes ranging from nano-to kilometer scale and from the Earth's surface to the mantle. Fluids enhance mineral reactions and melting, facilitate mass transport and change bulk physical rock properties such as density and rheology.

Modelling the interplay between rock deformation, fluid transport and reaction increases the understanding and the capability to predict fundamental geological processes which may also be relevant to societal problems such as oil and gas migration, $\mathrm{CO}_{2}$ capture and storage, geothermal energy, or ore genesis in hydrothermal systems. Therefore a variety of formulations and numerical models have been developed to simulate reactive transport in a porous medium (Steefel et al. 2005).

State-of the art reactive transport deformation models for metamorphic rocks commonly assume either incompressible solid or fluid leading to violation of mass conservation (Hetenyi 2014).

\section{Theoretical model}

Here, we present a next step in theoretical development of reactive-fluid flow models. The approach is based on mass conservation and porous flow and treats porosity and permeability evolution due to deformation and reaction. Minerals and fluid are compressible, variable pressure is used in calculating local equilibrium. Solid reactions are computed based on Gibbs minimization allowing for any possible reaction at each local equilibrium domain. Partitioning between fluid and solid based on solubility data is combined with diffusion and advection of chemical species leading to changes in composition at any location in the modelled domain thereby self-consistently accounting for local effective bulk composition which is then used in the calculation of thermodynamic equilibrium.

\section{Model setup}

An explicit finite difference numerical model is used to simulate reactive fluid flow on thin section and outcrop scale for three different settings 1 ) a serpentinite in a subduction zone (John et al. 2011) and 2) a metapelitic layered sequence during regional metamorphism and 3) metasomatic zoning due to injection of a felsic reactive agent in the Svartberget peridotite Western Gneiss Region, Norway (Vrijmoed et al. 2013).

For an un-deformed rock the initial fluid pressure rise results mainly from volume changes of reaction. Due to spatial compositional heterogeneity porosity generated by reactions varies locally. As metamorphic rocks are commonly under confinement the initial stages of reaction take place under near isochoric conditions. Therefore locally there will be a spontaneous spatial fluid pressure variation which will lead to flow. The magnitude of the pressure variations is estimated on the $\mu \mathrm{m}$-scale or outcrop scale using equilibrium thermodynamic calculations. 
Local bulk compositions are derived from 1) X-ray intensity maps on a $\mu$ m-scale, 2) synthetic layered metapelitic rock based on average metapelite composition and 3) from real observations documented by detailed outcrop studies and bulk rock analyses. The resulting compositional maps are used as initial condition to model the reactive fluid flow.

\section{Preliminary results and outlook}

The main results divide into 1) a set of 2D simulations that lead to veining patterns of porosity and dehydrated rocks (see Fig. 1) and 2) a set of 2D simulations that largely inherit the initial spatial compositional variation. We further investigate the effects of shearing on the reactive fluid flow and the resulting porosity and mineralogical patterns.

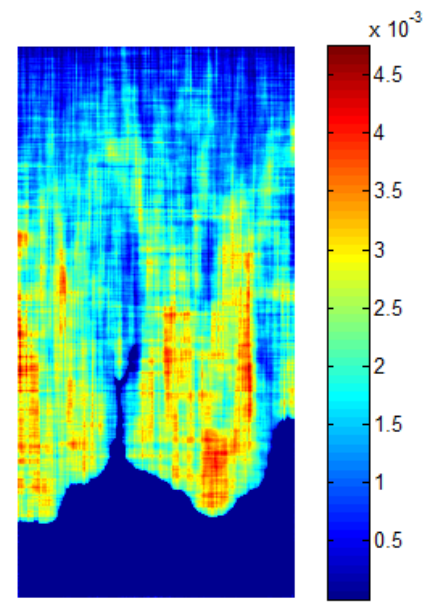

Fig. 1. Porosity distribution showing the development of veining patterns from an initially non-porous rock. Model was initialized with a porous zone $\left(1.5 \mathrm{e}^{-4}\right)$ at the top resulting from a dehydration reaction. A gradual change in composition varying from top to bottom underlies the model domain. Gravity is not included in this simulation as it is intended to show the effect on a thin section scale domain in which gravity may be assumed constant. (This simulation is for the case mass transport has negligible effect on the P-T conditions of the main reactions).

\section{Acknowledgements}

This work was supported by ERC starting grant 335577 to Lucie Tajcmanova.

\section{References}

Hetenyi G. (2014), To conserve or not to conserve (mass in numerical models). Terra Nova 26:372-376. doi: 10.1111/ter.12109

John T., Scambelluri M., Frische M., Barnes, J.D., Bach, W. (2011) Dehydration of subducting serpentinite: Implications for halogen mobility in subduction zones and the deep halogen cycle. Earth Planet Sci Lett 308:65-76. doi: 10.1016/j.epsl.2011.05.038

Steefel C., De Paolo D., Lichtner P. (2005) Reactive transport modeling: An essential tool and a new research approach for the Earth sciences. Earth Planet Sci Lett 240:539-558. doi: 10.1016/j.epsl.2005.09.017

Vrijmoed J. C., Austrheim H., John T., Hin, R.C., Corfu, F., Davies, G.R. (2013), Metasomatism in the Ultrahigh-pressure Svartberget Garnet-peridotite (Western Gneiss Region, Norway): 
Implications for the Transport of Crust-derived Fluids within the Mantle. J Petrol 54:1815-1848. doi: 10.1093/petrology/egt032 